\title{
PRODUÇÃO DE ÁCIDO LÁTICO A PARTIR DA CONVERSÃO HIDROTÉRMICA ALCALINA DA GLICERINA UTILIZANDO CATALISADORES HOMOGÊNEOS
}

\author{
A. K. O. RODRIGUES ${ }^{1}$, D. L. H. MAIA ${ }^{1}$, F. A. N. FERNANDES ${ }^{1}$ \\ ${ }^{1}$ Universidade Federal do Ceará, Departamento de Engenharia Química \\ E-mail para contato: k2_rodrigues@hotmail.com
}

\begin{abstract}
RESUMO - Devido ao aumento na produção de biodiesel há um excesso de glicerina no mercado. A conversão da glicerina em um produto com maior valor agregado para destinação ao mercado é vista de forma positiva. O ácido lático, que pode ser obtido a partir do glicerol, é um produto de grande interesse podendo ter múltiplas aplicações na indústria alimentícia, têxtil, cosmética, etc. Neste contexto, a síntese de ácido lático pela conversão hidrotérmica alcalina de glicerina pode indicar uma rota alternativa à rota fermentativa, visando adicionar um maior valor agregado à glicerina. $\mathrm{NaOH}$ e $\mathrm{KOH}$ foram usados como catalisadores. Temperatura $\left(240-280^{\circ} \mathrm{C}\right)$, pressão $(28-43$ bar), razão volumétrica de água/glicerina (0,8-30,9), razão molar catalisador/glicerol $(0,01-1,02)$ e tempo total de reação (3-4 horas) foram as variáveis estudadas. A temperatura e a razão volumétrica de água/glicerina foram as variáveis de maior influência. O maior rendimento obtido foi de $28,1 \%$, a $255,0^{\circ} \mathrm{C}$ e a 32,1 bar, em um tempo total de reação de $3 \mathrm{~h}$, a partir de uma solução de razão volumétrica água/glicerina igual a 0,8 e razão molar $\mathrm{KOH} /$ glicerol igual a 0,03 . A partir deste resultado, processo hidrotérmico pode ser visto como sendo um processo promissor para agregar valor à glicerina.
\end{abstract}

\section{INTRODUÇÃO}

A utilização de fontes alternativas de energia é uma das grandes prioridades atuais, que surge para contornar os graves problemas ocasionados pelo desenvolvimento tecnológico. Neste sentido, o biodiesel surge como um combustível alternativo ao petróleo. Porém, a produção de biodiesel gera uma grande quantidade de glicerina (10\% em massa) que é considerada um subproduto indesejado (Dasari et al., 2005).

Para aumentar o valor de mercado da glicerina, faz-se necessário convertê-la em outros produtos químicos, como por exemplo, em ácido lático, que está se tornando cada vez mais importante. Atualmente, o ácido lático é usado como um precursor de solventes verdes, tais como, o lactato de etila e na síntese de poli(ácido lático), PLA, que é cada vez mais utilizado para a produção de embalagens biodegradáveis. $\mathrm{O}$ ácido lático possui múltiplas aplicações nas indústrias de alimentos, têxtil, de couro, cosmética, química e farmacêutica. No entanto, o uso de ácido lático é limitado pelo seu preço elevado, pois $95 \%$ do ácido lático obtido, a nível mundial, é produzido por via fermentativa (He et al., 2008).

O processo hidrotérmico é um processo promissor para a conversão de glicerina em produtos químicos de alto valor agregado (Kishida et al., 2009). A reação hidrotérmica ocorre em meio aquoso e envolve a aplicação de calor sob pressão. 
A reação global da conversão hidrotérmica alcalina da glicerina em ácido lático é representada pela Equação 1:

$$
\mathrm{C}_{3} \mathrm{H}_{8} \mathrm{O}_{3}+\mathrm{NaOH} \rightarrow \mathrm{C}_{3} \mathrm{H}_{5} \mathrm{O}_{3} \mathrm{Na}+\mathrm{H}_{2} \uparrow+\mathrm{H}_{2} \mathrm{O}
$$

Nos estudos realizados por Kishida et al. (2009), Ramírez-López et al. (2010) e Fang et al. (2011) pequenas concentrações de glicerina (0,3 a 3,5 Molar) foram usadas durante processo hidrotérmico para convertê-la em ácido lático. Tais estudos mostram que conversões maiores que $85 \%$ foram obtidas. Contudo, os valores de produtividade obtidos eram muito baixos (menor que $20 \mathrm{~g} / \mathrm{L} . \mathrm{h}$ ).

O presente estudo teve como objetivo utilizar o processo hidrotérmico como potencial para produção de ácido lático, em substituição ao processo fermentativo que é, atualmente, utilizado para a produção deste produto.

O principal caráter inovativo deste estudo consistiu no uso de significativas concentrações de glicerol (0,51 a 17,1 Molar), visando obter produtividades elevadas. As concentrações de glicerol utilizadas no presente estudo foram bastante superiores às concentrações de glicerol usadas nos estudos realizados por Kishida et al. (2009), RamírezLópez et al. (2010) e Fang et al. (2011).

Neste contexto, a síntese de ácido lático pela conversão hidrotérmica alcalina de glicerina pode indicar uma rota alternativa para adicionar um maior valor agregado à glicerina.

\section{MATERIAIS E MÉTODOS}

\subsection{Materiais}

Glicerina PA (Synth - Brasil), glicerina bruta (Petrobras Biocombustível, Quixadá-CE), água destilada, hidróxido de sódio PA (Dinâmica - Brasil), hidróxido de potássio PA (Vetec Brasil), metanol PA (Synth - Brasil) e ácido sulfúrico PA (Synth - Brasil) foram utilizados como reagentes.

As reações foram realizadas em um reator (Metalquim Ind. - Brasil) de aço inoxidável de alta pressão, de $350 \mathrm{~mL}$, equipado com dispositivos de medição de temperatura e pressão, além de um agitador magnético, um aquecedor e um controlador de temperatura (Metalquim Ind. - Brasil).

Ar sintético, $\mathrm{N}_{2}, \mathrm{He}$, e $\mathrm{H}_{2}$, todos eles com $99,99 \%$ de pureza, produzidos pela White Martins (Brasil), foram utilizados no cromatografo gasoso (Trace ${ }^{\mathrm{TM}}$ Ultra - Thermo Scientific, EUA). Além disso, o $\mathrm{N}_{2}$ também foi utilizado no primeiro planejamento experimental a fim de aumentar a pressão no interior do reator, para que a pressão interna resultante admitisse valores superiores a 28 bar e, portanto, auxiliasse na conversão da glicerina.

\subsection{Procedimento Experimental}

A conversão hidrotérmica do glicerol em ácido lático foi realizada a temperatura de 240-280 ${ }^{\circ} \mathrm{C}$, pressão de 29-43 bar, razões volumétricas de água/glicerol de 0,8-30,9, razão 
molar catalisador/glicerol de 0,01-1,02 e tempo de reação de 3 horas. Os catalisadores utilizados foram o hidróxido de sódio $(\mathrm{NaOH})$ e o hidróxido de potássio $(\mathrm{KOH})$.

As reações iniciais são caracterizadas pelo uso de uma baixa concentração de glicerina $(0,51 \mathrm{M})$ e as reações posteriores são caracterizadas pelo uso de uma maior concentração de glicerina (10,9 a 17,1 M), a fim de obter maiores níveis de concentração e produtividade de ácido lático.

\subsection{Análise das Amostras}

As amostras obtidas ao final de cada reação foram analisadas em um cromatógrafo gasoso (CG - Trace ${ }^{\mathrm{TM}}$ Ultra - Thermo Scientific, EUA), utilizando o detector FID (flame ionization detector ou detector de ionização de chama) e coluna capilar OV-1 (30 m de comprimento x $0,25 \mathrm{~mm}$ de diâmetro interno x 0,25 $\mu \mathrm{m}$ de espessura de filme).

O ácido lático foi convertido em lactato de metila, antes da análise no CG-FID, para uma melhor análise dos resultados, pois a sensibilidade do CG-FID é muito baixa, no que diz respeito à identificação e quantificação de ácido lático. Assim, o preparo das amostras analisadas no cromatógrafo gasoso (CG-FID) consistiu, inicialmente, na mistura de $10 \mathrm{~mL}$ da solução de ácido lático com 6 gotas de ácido sulfúrico PA e $25 \mathrm{~mL}$ de metanol PA e, posteriormente, foi retirada uma alíquota de $50 \mu \mathrm{L}$ desta mistura inicial e a esta foram adicionados $1,5 \mathrm{~mL}$ de metanol PA.

As equações abaixo descrevem, respectivamente, a reação hidrotérmica alcalina, utilizando $\mathrm{NaOH}$ como catalisador, a hidrólise pelo uso de $\mathrm{H}_{2} \mathrm{SO}_{4}$ e a esterificação pelo uso de $\mathrm{CH}_{3} \mathrm{OH}$.

$$
\begin{aligned}
& \mathrm{CH}_{3} \mathrm{CHOHCOOH}+\mathrm{NaOH} \rightarrow\left(\mathrm{CH}_{3} \mathrm{CHOHCOO}^{-}\right) \mathrm{Na}^{+}+\mathrm{H}_{2} \uparrow+\mathrm{H}_{2} \mathrm{O} \\
& \text { Ácido Lático Lactato de Sódio } \\
& \left(\mathrm{CH}_{3} \mathrm{CHOHCOO}^{-}\right) \mathrm{Na}^{+}+\mathrm{H}_{2} \mathrm{SO}_{4} \rightarrow \mathrm{CH}_{3} \mathrm{CHOHCOOH}+\mathrm{Na}_{2} \mathrm{SO}_{4} \\
& \text { Lactato de Sódio Ácido Lático } \\
& \mathrm{CH}_{3} \mathrm{CHOHCOOH}+\mathrm{CH}_{3} \mathrm{OH} \rightarrow \mathrm{CH}_{3} \mathrm{CHOHCOOCH}+\mathrm{H}_{2} \mathrm{O} \\
& \text { Ácido Lático Lactato de Metila }
\end{aligned}
$$

As amostras $(1 \mu \mathrm{L})$ foram injetadas a uma temperatura do forno de $150^{\circ} \mathrm{C}$. A temperatura do injetor e do detector foi de $250^{\circ} \mathrm{C}$.

Padrões analíticos de lactato de metila (85\%) foram utilizados como referência nas análises no CG-FID e uma curva de calibração foi estabelecida para inferir a concentração de cada amostra obtida nos experimentos.

\section{RESULTADOS E DISCUSSÕES}

Nos ensaios iniciais (Tabelas 1 e 2) o hidróxido de sódio $(\mathrm{NaOH})$ foi utilizado como catalisador da reação de conversão. Uma pequena quantidade de glicerina PA foi utilizada e a razão molar $\mathrm{NaOH} /$ glicerina no meio reacional variou entre 0,04-1,02. Amostras foram analisadas no $\mathrm{CG}$. 
A Tabela 1 exibe as combinações iniciais usadas no planejamento experimental inicial, além dos valores referentes à temperatura $(\mathrm{T})$, pressão $(\mathrm{P})$, rendimento, concentração do ácido lático e produtividade.

Tabela 1 - Rendimentos, concentrações e produtividades obtidas no planejamento experimental 1.

\begin{tabular}{cccccccc}
\hline Amostras & $\begin{array}{c}\text { Razão molar de } \\
\text { NaOH/glicerina }\end{array}$ & $\begin{array}{c}\text { Razão } \\
\text { volumétrica de } \\
\text { água/glicerina }\end{array}$ & $\begin{array}{c}\mathrm{T} \\
\left({ }^{\circ} \mathrm{C}\right)\end{array}$ & $\begin{array}{c}\mathrm{P} \\
(\mathrm{bar})\end{array}$ & $\begin{array}{c}\text { Rendimento } \\
(\%)\end{array}$ & $\begin{array}{c}\mathrm{C} \\
(\mathrm{g} / \mathrm{L})\end{array}$ & $\begin{array}{c}\text { Produtividade } \\
(\mathrm{g} / \mathrm{L} . \mathrm{h})\end{array}$ \\
\hline Teste 1 & 0,44 & 30,9 & 247 & 37,1 & 67,6 & 26,3 & 8,8 \\
Teste 2 & 0,44 & 30,9 & 250 & 43,0 & 76,4 & 29,7 & 9,9 \\
Teste 3 & 0,44 & 26,9 & 240 & 32,5 & 51,1 & 22,8 & 7,6 \\
Teste 4 & 0,73 & 30,8 & 250 & 43,0 & 51,3 & 20,0 & 6,7 \\
Teste 5 & 0,73 & 30,8 & 252 & 42,0 & 53,4 & 20,8 & 6,9 \\
Teste 6 & 0,73 & 26,8 & 245 & 40,0 & 50,2 & 22,5 & 7,5 \\
Teste 7 & 1,02 & 30,7 & 241 & 32,3 & 47,1 & 18,4 & 6,1 \\
Teste 8 & 1,02 & 30,7 & 244 & 34,7 & 50,2 & 19,6 & 6,5 \\
Teste 9 & 1,02 & 26,7 & 240 & 30,4 & 43,4 & 19,5 & 6,5 \\
\hline
\end{tabular}

Na Tabela 1 são exibidos os valores de rendimento, concentração e produtividade dos respectivos testes. Os rendimentos obtidos são consideráveis, uma vez que a maioria se mostra superior a $50 \%$.

Assim, comparando as condições operacionais, em termos de temperatura e pressão utilizadas estudo realizado por Kishida et al. (2009), com as condições operacionais utilizadas no presente estudo, verifica-se que no estudo de Kishida et al. (2009) as condições operacionais foram mais severas e, portanto, houve um maior consumo energético, o qual foi necessário para proporcionar o aquecimento das amostras a $300^{\circ} \mathrm{C}$. Tal severidade, em termos de temperatura e pressão foi primordial para que um rendimento de $90 \%$ fosse alcançado.

Além disso, no estudo realizado por Kishida et al. (2009), a concentração de ácido lático correspondente ao rendimento de $90 \%$ foi de $26,7 \mathrm{~g} / \mathrm{L}$ e a produtividade, considerando o tempo total de reação, foi de $8,9 \mathrm{~g} / \mathrm{L} . \mathrm{h}$.

De um modo geral, a produtividade obtida nos ensaios deste planejamento experimental é muito baixa. Isso pode ser explicado pelo fato de que uma quantidade muito pequena de glicerina foi utilizada no meio reacional. Além disso, vale frisar que as condições operacionais utilizadas não admitiram uma significativa severidade, em termos de temperatura e pressão, se comparada com as condições usadas no estudo de Kishida et al. (2009).

No primeiro planejamento experimental, os valores de rendimento foram bastante satisfatórios, contudo os valores de produtividade foram baixíssimos, uma vez que a concentração de glicerina usada, no meio reacional, também foi muito baixa (0,51 M). Assim, foi realizada uma segunda bateria de ensaios experimentais na qual uma maior concentração de glicerina $(13,7 \mathrm{M})$ bruta foi utilizada. 
A Tabela 2 exibe os respectivos valores de rendimento, concentração e produtividade correspondentes aos ensaios do segundo planejamento experimental, o qual teve com base o uso de glicerina proveniente do processo de transesterificação do biodiesel e $\mathrm{NaOH}$ como catalisador.

Tabela 2 - Rendimentos, concentrações e produtividades obtidas no planejamento experimental 2.

\begin{tabular}{cccccccc}
\hline Amostras & $\begin{array}{c}\text { Razão molar de } \\
\text { NaOH/glicerol }\end{array}$ & $\begin{array}{c}\text { Razão } \\
\text { volumétrica de } \\
\text { água/glicerol }\end{array}$ & $\mathrm{T}\left({ }^{\circ} \mathrm{C}\right)$ & $\begin{array}{c}\mathrm{P} \\
(\mathrm{bar})\end{array}$ & $\begin{array}{c}\text { Rendimento } \\
(\%)\end{array}$ & $\begin{array}{c}\mathrm{C} \\
(\mathrm{g} / \mathrm{L})\end{array}$ & $\begin{array}{c}\text { Produtividade } \\
(\mathrm{g} / \mathrm{L} . \mathrm{h})\end{array}$ \\
\hline Teste 10 & 0,04 & 1 & 260,8 & 29,7 & 19,0 & 91,2 & 30,4 \\
Teste 11 & 0,07 & 1 & 260,7 & 30,1 & 6,5 & 31,4 & 10,5 \\
Teste 12 & 0,11 & 1 & 260,2 & 30,3 & 2,2 & 10,3 & 3,4 \\
Teste 13 & 0,04 & 1 & 270,2 & 29,9 & 18,0 & 86,3 & 28,8 \\
Teste 14 & 0,07 & 1 & 270,5 & 31,2 & 8,9 & 42,7 & 14,2 \\
Teste 15 & 0,11 & 1 & 270,3 & 31,1 & 2,1 & 10,1 & 3,4 \\
Teste 16 & 0,04 & 1 & 280,8 & 33,4 & 16,9 & 81,3 & 27,1 \\
Teste 17 & 0,07 & 1 & 280,2 & 32,8 & 14,3 & 68,5 & 22,8 \\
Teste 18 & 0,11 & 1 & 280,4 & 33,1 & 2,0 & 9,7 & 3,2 \\
\hline
\end{tabular}

A partir da Tabela 2, observa-se que os rendimentos correspondentes a este planejamento foram baixos, se comparados com os rendimentos obtidos no primeiro planejamento experimental. Porém, os valores de concentração e produtividade foram superiores, uma vez que a quantidade de glicerol usada no segundo planejamento experimental foi bem superior à quantidade de glicerol usada no primeiro planejamento experimental.

Além disso, praticamente, não foram observadas alterações nos rendimentos para os testes envolvendo a mesma massa de catalisador, a mesma razão água/glicerol, o mesmo tempo total de reação $(3 \mathrm{~h})$ e diferentes temperaturas. Como consequência disso, também não foram observadas alterações nos valores de concentração e produtividade. Logo, verifica-se que o aumento da temperatura, neste caso, não implicou no aumento da conversão de glicerol em ácido lático.

Comparando o Teste 2, cuja razão molar de $\mathrm{NaOH} /$ glicerol usada foi de 0,44 , razão volumétrica água/glicerina igual a 30,9 e temperatura de $250^{\circ} \mathrm{C}$, com os Testes 10,13 e 16 , cuja razão molar de $\mathrm{NaOH} /$ glicerol usada foi de 0,04 , razão volumétrica água/glicerina igual 1 e temperaturas de $260,8^{\circ} \mathrm{C}, 270,2^{\circ} \mathrm{C}$ e $280,8^{\circ} \mathrm{C}$, respectivamente, nota-se que o Teste 2 apresentou o maior rendimento, enquanto que os Testes 10,13 e 16 apresentaram maior concentração e produtividade, já que a concentração de glicerol presente no meio reacional foi maior nos Testes 10, 13 e 16 .

Constata-se, também, que a glicerina bruta não necessita de um refinamento antes de ser utilizada no processo hidrotérmico alcalino. Faz-se necessário, apenas, uma filtração da glicerina bruta para remover materiais suspensos contidos nesta glicerina. 
Kishida et al. (2009) realizaram um estudo no qual verificou-se um melhor desempenho do $\mathrm{KOH}$ frente ao $\mathrm{NaOH}$, nas reações de conversão hidrotérmica de glicerol em ácido lático. Sendo assim, um terceiro planejamento experimental foi traçado, tomando como referência o uso do $\mathrm{KOH}$ como catalisador. Além disso, optou-se em trabalhar numa faixa de temperatura inferior a da segunda bateria de experimentos.

O terceiro planejamento experimental teve como objetivo avaliar a contribuição das novas condições operacionais em relação ao rendimento obtido. A concentração de glicerina bruta utilizada no meio reacional variou entre 10,9 e 17,1 M. As amostras obtidas ao final de cada reação também foram analisadas via CG.

A Tabela 3 exibe os respectivos rendimentos correspondentes aos ensaios referentes ao terceiro planejamento experimental, o qual teve com base o uso do $\mathrm{KOH}$ como catalisador.

Tabela 3 - Rendimentos, concentrações e produtividades obtidas no planejamento experimental 3.

\begin{tabular}{cccccccc}
\hline Amostras & $\begin{array}{c}\text { Razão molar de } \\
\text { KOH/glicerol }\end{array}$ & $\begin{array}{c}\text { Razão } \\
\text { volumétrica de } \\
\text { água/glicerol }\end{array}$ & $\mathrm{T}\left({ }^{\circ} \mathrm{C}\right)$ & $\mathrm{P}(\mathrm{bar})$ & $\begin{array}{c}\text { Rendimento } \\
(\%)\end{array}$ & $\mathrm{C}(\mathrm{g} / \mathrm{L})$ & $\begin{array}{c}\text { Produtividade } \\
(\mathrm{g} / \mathrm{L} . \mathrm{h})\end{array}$ \\
\hline Teste 19 & 0,03 & 1,25 & 252,3 & 31,9 & 2,7 & 13,0 & 4,3 \\
Teste 20 & 0,03 & 0,8 & 255,0 & 32,1 & 28,1 & 134,8 & 44,9 \\
Teste 21 & 0,03 & 1 & 254,8 & 31,6 & 19,5 & 93,8 & 31,3 \\
Teste 22 & 0,05 & 1,25 & 251,2 & 28,3 & 2,8 & 13,5 & 4,5 \\
Teste 23 & 0,04 & 0,8 & 256,5 & 30,0 & 21,3 & 102,2 & 34,1 \\
Teste 24 & 0,04 & 1 & 254,6 & 30,1 & 18,1 & 87,0 & 29,0 \\
Teste 25 & 0,07 & 1,25 & 249,6 & 28,5 & 3,7 & 18,0 & 6,0 \\
Teste 26 & 0,05 & 0,8 & 260,0 & 30,1 & 19,6 & 93,9 & 31,3 \\
Teste 27 & 0,05 & 1 & 252,1 & 29,3 & 16,8 & 80,8 & 26,2 \\
\hline
\end{tabular}

A partir da Tabela 3 é possível observar que os maiores rendimentos foram obtidos nos ensaios cuja razão volumétrica água/glicerol foi a menor utilizada - igual a 0,8 . Ou seja, para a mesma massa de catalisador utilizada e mesmo tempo total de reação $(3 \mathrm{~h})$, os maiores rendimentos foram obtidos quando a quantidade de água presente no meio reacional se mostrou a menor entre as proporções volumétricas utilizadas.

Comparando os Testes 10, 13 e 16 com o Teste 21, é possível verificar que o $\mathrm{KOH}$ apresenta um melhor desempenho frente ao $\mathrm{NaOH}$, como já havia sido observado no estudo realizado por Kishida et al. (2009).

Um quarto planejamento experimental foi realizado com o intuito de avaliar a influência do tempo de reação frente ao rendimento obtido.

$\mathrm{Na}$ quarta bateria de ensaios a quantidade de catalisador - $\mathrm{KOH}$ - utilizada foi de apenas $1 \mathrm{~g}$, razão volumétrica água/glicerol igual a 0,8, razão molar $\mathrm{KOH} / \mathrm{glicerol}$ igual a 0,01 e tempo total de reação de 3 e 4 horas. 
A Tabela 4 exibe os respectivos rendimentos correspondentes aos ensaios referentes ao quarto planejamento experimental.

Tabela 4 - Rendimentos, concentrações e produtividades obtidas no planejamento experimental 4.

\begin{tabular}{cccccccc}
\hline Amostras & $\begin{array}{c}\text { Razão molar de } \\
\text { KOH/glicerol }\end{array}$ & $\begin{array}{c}\text { Tempo de } \\
\text { reação }(\mathrm{h})\end{array}$ & $\mathrm{T}\left({ }^{\circ} \mathrm{C}\right)$ & $\mathrm{P}($ bar $)$ & $\begin{array}{c}\text { Rendimento } \\
(\%)\end{array}$ & $\begin{array}{c}\mathrm{C} \\
(\mathrm{g} / \mathrm{L})\end{array}$ & $\begin{array}{c}\text { Produtividade } \\
(\mathrm{g} / \mathrm{L} . \mathrm{h})\end{array}$ \\
\hline Teste 28 & 0,01 & 3 & 245,3 & 28,7 & 27,7 & 133,0 & 44,3 \\
Teste 29 & 0,01 & 4 & 253,0 & 30,3 & 27,6 & 132,8 & 33,2 \\
\hline
\end{tabular}

A partir da Tabela 4 verifica-se que um maior tempo reacional não contribuiu para que um rendimento maior fosse obtido e, portanto, não há necessidade de gastar mais energia com o aquecimento da mistura reacional, já que não houve aumento no valor do percentual correspondente ao rendimento do ácido lático produzido.

No Teste 28 , os valores correspondes à concentração $(133,0 \mathrm{~g} / \mathrm{L})$ e produtividade $(44,3$ g/L.h), mostram-se significativamente superiores se comparados com valores de concentração e produtividade do ácido lático obtido por via fermentativa. Por exemplo, nos estudos realizados por Ryu et al. (2005) e Parajó et al. (2005), nos quais a produção de ácido lático foi realizada a partir da fermentação do trigo por Enterococcus faecalis e do resíduo de papelão por Lactobacillus coryniformis, respectivamente, os valores correspondentes à concentração e à produtividade do ácido lático foram de $102,7 \mathrm{~g} / \mathrm{L}$ e $23,1 \mathrm{~g} / \mathrm{L} ;$ e $3,8 \mathrm{~g} / \mathrm{L} \cdot \mathrm{h}$ e 0,5 g/L.h, respectivamente.

Nos trabalhos realizados por Dong et al. (2013), Labidi et al. (2012) e Kishida et al. (2009), em que a conversão hidrotérmica do glicerol em ácido lático foi estudada, os valores de rendimentos, concentrações e produtividades obtidos foram de 89,6\%, 44,76\% e 90\%; 12,6 g/L, 7,38 g/L e 26,7 g/L; 6,3 g/L.h, 14,76 g/L.h e 8,9 g/L.h, respectivamente. Logo, comparando os valores de rendimento, concentração e produtividade obtidos nos estudos de Dong et al. (2013), Labidi et al. (2012) e Kishida et al. (2009) com os valores obtidos nos Testes 20 e 28 do presente estudo, verifica-se que os rendimentos dos Testes 20 e 28 são muito baixos, porém os valores de concentração e produtividade são bastante elevados, o que reflete uma vantagem.

\section{CONCLUSÕES}

No presente estudo, observou-se que as variáveis que exercem influência sobre a conversão do glicerol em ácido lático são a razão volumétrica de água/glicerol, massa de catalisador presente no meio reacional e a temperatura.

$\mathrm{O}$ desempenho do $\mathrm{KOH}$ frente ao $\mathrm{NaOH}$ se mostrou superior. $\mathrm{O}$ maior rendimento obtido foi de $28,1 \%$, a $255,0^{\circ} \mathrm{C}$ e a 32,1 bar, em um tempo reacional de $3 \mathrm{~h}$, partindo de uma solução cuja razão volumétrica água/glicerol foi a menor utilizada - igual a 0,8 - e cuja massa de catalisador $(\mathrm{KOH})$ foi de $2 \mathrm{~g}$.

As condições operacionais utilizadas no presente estudo foram bastante satisfatórias no que tange à concentração e à produtividade de ácido lático e, portanto, o processo hidrotérmico pode ser, de fato, considerado um processo promissor, capaz de adicionar um maior valor agregado à glicerina. 


\section{REFERÊNCIAS}

DASARI, M. A.; KIATSIMKUL, P. P.; SUTTERLIN, W. R.; SUPPES, G. J. Low-pressure hydrogenolysis of glycerol to propylene glycol. Appl. Catal. A: Gen., 281, p.225, 2005.

DONG, W. S.; WANG, F. F.; LIU, C. L. Highly efficient production of lactic acid from cellulose using lanthanide triflate catalysts. Green Chemistry, 15, 2091-2095, 2013.

FANG, Z.; LONG, Y. D.; GUO, F.; TIAN, X. F.; JIANG, L. Q.; ZHANG, F. Production of biodiesel and lactic acid from rapeseed oil using sodium silicate as catalyst. Bioresource Technology, 102, 6884-6886, 2011.

HE, W.; LI, G.; KONG, L.; WANG, H.; HUANG, J.; XU, J. Application of hydrothermal reaction in resource recovery of organic wastes. Resources, Conservation and Recycling, v. 52, n.5, p. 691-699, 2008.

KISHIDA, H.; SHEN, Z.; JIN, F.; ZHANG, Y.; WU, B.; KISHITA, A.; TOHJ, K. Effect of Alkaline Catalysts on Hydrothermal Conversion of Glycerin into Lactic Acid. Ind. Eng. Chem. Res., 48 (19), p. 8920-8925, 2009.

LABIDI, J.; SANCHEZ, C.; EGUEES, I.; GARCIA, A.; LLANO-PONTE, R. Lactic acid production by alcaline hydrothermal treatment of corn cobs. Chem. Eng. J., 181, 655-660, 2012.

PARAJÓ, J. C.; YÁÑEZ, R.; ALONSO, J. L. D-lactic acid production from waste cardboard, J. Chem. Technol. Biotechnol. 80, 76-84, 2005.

RAMÍEZ-LÓPEZ, C. A.; OCHOA-GÓMEZ, J. R.; FERNÁNDEZ-SANTOS, M.; GÓMEZJIMÉNEZ-ABERASTURI, O.; ALONSO-VICARIO, A.; TORRECILLA-SORIA, J. Synthesis of Lactic Acid by Alkaline Hydrothermal Conversion of Glycerol at High Glycerol Concentration. Ind. Eng. Chem. Res. 49, 6270-6278, 2010.

RYU, H. W.; OH, H.; WEE, Y. J.; YUN, J. S.; HAN, S. H.; JUNG, S. Lactic acid production from agricultural resources as cheap raw materials. Bioresour. Technol. 96, 1492-1498, 2005. 